\title{
Prognostic value of pretreatment and recovery duration of cranial nerve palsy in nasopharyngeal carcinoma
}

\author{
Hao-Yuan Mo ${ }^{1,2 \dagger}$, Rui Sun ${ }^{1,2+}$, Jian Sun ${ }^{1,3}$, Qing Zhang ${ }^{4}$, Wen-Jin Huang ${ }^{5}$, Yan-Xian Li ${ }^{1,2}$, Jing Yang ${ }^{1,2}$ \\ and Hai-Qiang Mai ${ }^{1,2^{*}}$
}

\begin{abstract}
Background: The purpose of this study was to evaluate the prognostic value of cranial nerve (CN) palsy in nasopharyngeal carcinoma (NPC) patients.

Methods: A retrospective analysis was performed on CN involvement using medical records of 178 consecutive patients with histologically diagnosed, non-disseminated NPC.

Results: In 178 NPC patients with CN palsy, the 5-year survival rates were as follows: overall survival (OS), 61.0\%; disease-specific survival (DSS), 69.6\%; local relapse-free survival (LRFS), 75.2\%; distant metastasis-free survival (DMFS), 73.4\%; and disease-free survival (DFS), 55.3\%. Significant differences were observed in the 5-year OS rates between patients with single and multiple CN palsy $(69.8 \%$ vs. $54.3 \%$; $P=0.033)$ and the OS rates between patients with different pretreatment durations $(68.7 \%$ vs. $43.3 \%, P=0.007)$. However, no significant differences were observed in OS, DSS, LRFS and DFS rates between patients with upper and lower $C N$ palsy $(P=0.581, P=0.792, P=0.729$ and $P=0.212$, respectively). The results showed that recovery duration was an independent prognostic factor for OS $(H R=2.485 ; P<0.001)$, DSS $(H R=2.065 ; P=0.016)$, LRFS $(H R=3.051 ; P=0.001)$ and DFS $(H R=2.440 ; P<0.001)$.

Conclusions: Recovery duration is an independent prognostic factor for NPC patients with CN palsy and is related to recurrence, which leads to poor survival. Recovery duration requires close surveillance and different treatment regimens.
\end{abstract}

Keywords: Nasopharyngeal carcinoma, Cranial nerve involvement, Pretreatment duration, Recovery duration, Prognosis

\section{Background}

Cranial nerve $(\mathrm{CN})$ palsy in nasopharyngeal carcinoma (NPC) is the result of an adjacent extension of the primary tumor. $\mathrm{CN}$ palsy occurs in 8.0 to $12.4 \%$ of cases [1]. Cranial nerve involvement is considered an unfavourable prognostic factor for NPC patients, and those with $\mathrm{CN}$ palsy have been classified as $\mathrm{T} 4$ according to

\footnotetext{
* Correspondence: maihq@mail.sysu.edu.cn

${ }^{\dagger}$ Equal contributors

${ }^{1}$ State Key Laboratory of Oncology in South China, Sun Yat-sen University Cancer Center, No, 651 Dongfeng Road East, Guangzhou 510060, People's Republic of China

${ }^{2}$ Department of Nasopharyngeal Carcinoma, Sun Yat-sen University Cancer Center, 651 Dongfeng Road East, Guangzhou 510060, People's Republic of China

Full list of author information is available at the end of the article
}

the staging system of NPC in the seventh edition of the American Joint Committee on Cancer (AJCC) [2]. The patients with extensive $\mathrm{CN}$ palsy had worse survival rates than those with only upper $\mathrm{CN}$ or lower $\mathrm{CN}$ involvement [3]. Moreover, the patients who recovered from $\mathrm{CN}$ palsy had better survival rates than those who did not. However, no recent research has indicated the prognostic value of the pretreatment or recovery durations for NPC patients with CN palsy.

The diagnosis of $\mathrm{CN}$ palsy mostly depends on clinical symptoms and a physical examination, and magnetic resonance imaging (MRI) has proven to be an important tool for defining CN involvement in NPC [4-6]. However, $\mathrm{CN}$ palsy frequently accompanies skull-base invasion in upper $\mathrm{CN}$ palsy and carotid sheath erosion in

\section{Biomed Central}


lower $\mathrm{CN}$ palsy. Skull-base invasion can approach from basicranial to intracranial or orbital sites, and carotid sheath erosion will extracranially involve lower $\mathrm{CN}$ palsy. Previous studies have focussed only on survival rates and imaging diagnoses in NPC patients with and without $\mathrm{CN}$ palsy, and those studies did not indicate the prognostic value of the pretreatment or recovery durations in NPC patients with CN palsy.

The National Comprehensive Cancer Network (NCCN) has recommended concurrent chemoradiotherapy plus adjuvant or induction chemotherapy as the standard treatment for patients with $\mathrm{CN}$ palsy. However, it is important to understand whether similar chemotherapy cycles and radiotherapy doses benefit NPC patients with $\mathrm{CN}$ palsy with different pretreatment and recovery durations. Hence, research on the prognostic value of the pretreatment and recovery durations of NPC patients with $\mathrm{CN}$ palsy was performed with a large sample size. Because the pretreatment program design is very important, in this study, we aimed to grade the pretreatment and recovery durations of NPC patients with CN palsy and to precisely judge patient prognosis. The results of this study may help to develop individualised treatment for NPC patients [7].

\section{Methods}

\section{Patients}

We reviewed the records of consecutive NPC patients referred to our center between January 1, 2002, and December 31, 2003. During the study period, there were 1,892 primary NPC patients who received radiation therapy in our institution. Of these patients, we identified 178 NPC patients with CN palsy $(9.4 \% ; 178 / 1892)$ who had completed radical treatment during the study period. All clinical records were reviewed by the authors. Of these patients, 145 patients were male, and 33 were female (a male-to-female ratio of 4.4:1). The patients had biopsy-proven NPC without distant metastases and were treated radically with radiation. The median age of the 145 male patients was 47.7 years (range, 1874 years), and the median age of the 33 female patients was 45.3 years (range, $21-67$ years).

The CN palsy diagnosis was mainly based on clinical symptoms and a physical examination. At histologic examination, 174 of the 178 patients $(97.8 \%)$ were classified as having type II or III disease based on the World Health Organization criteria. Three patients (1.7\%) were classified as having type I disease; the remaining patient (0.5\%) had an adenocarcinoma. All patients underwent a pretreatment evaluation that included a complete patient history, physical and neurologic examinations, haematological and biochemical profiles, MR imaging of the neck and nasopharynx, chest radiography and abdominal ultrasonography. Medical records were retrospectively analysed, and all patients were staged according to the staging system of the seventh edition of the American Joint Committee on Cancer. The stage distribution for the patients was as follows: $10.1 \%$ of patients (18/178) belonged to clinical stage IVb with N3 classification; other patients belonged to clinical stage IVa with T4 classification, including $16.3 \%$ of patients (29/178) classified as N0; $51.7 \%(92 / 178)$ as N1; and $21.9 \%(39 / 178)$ as N2. Table 1 shows the characteristics of all patients.

\section{Treatment}

All patients were treated with radical radiotherapy. The details of the radiation therapy techniques used at the Cancer Center of Sun Yat-sen University have been described previously [8-10]. In brief, most patients (146/ $178 ; 82.0 \%)$ were treated with conventional techniques, $13.5 \%(24 / 178)$ received intensity-modulated radiation therapy (IMRT) and 4.5\% (8/178) were administered 3dimensional conformal radiation therapy (3D-CRT).

A total of 44 of 178 patients (24.7\%) were treated with radiotherapy only. A total of 134 of 178 patients (75.3\%) received chemotherapy, including various regimens of concurrent chemotherapy combined with either induction chemotherapy or adjuvant chemotherapy in conjunction with a platinum-based therapeutic clinical trial. Deviations from the guidelines were due to advanced

Table 1 Patient characteristics $(n=178)$

\begin{tabular}{|c|c|c|}
\hline $\begin{array}{l}\text { Characteristics } \\
\text { Age }\end{array}$ & $\begin{array}{c}\begin{array}{c}\text { Number } \\
\text { Median: } 46.5\end{array} \\
\text { (Range, 18-74) }\end{array}$ & Percentage (\%) \\
\hline \multicolumn{3}{|l|}{ Sex } \\
\hline Male & 145 & 81.5 \\
\hline Female & 33 & 18.5 \\
\hline \multicolumn{3}{|l|}{ Biopsy (WHO) } \\
\hline I & 3 & 1.7 \\
\hline$\|+1\|$ & 174 & 97.8 \\
\hline Adenocarcinoma & 1 & 0.5 \\
\hline \multicolumn{3}{|l|}{ N stage ${ }^{*}$} \\
\hline NO & 29 & 16.3 \\
\hline N1 & 92 & 51.7 \\
\hline N2 & 39 & 21.9 \\
\hline N3 & 18 & 10.1 \\
\hline \multicolumn{3}{|l|}{ Radiotherapy } \\
\hline 2D-CRT & 146 & 82.0 \\
\hline IMRT & 24 & 13.5 \\
\hline 3D-CRT & 8 & 4.5 \\
\hline \multicolumn{3}{|l|}{ Chemotherapy } \\
\hline No & 44 & 24.7 \\
\hline Yes & 134 & 75.3 \\
\hline
\end{tabular}

§According to the AJCC seventh edition. 
age, heart disease, severe diabetes, or inadequate renal or hepatic function, which suggested intolerance to chemotherapy. When possible, salvage treatments, such as neck dissection and chemotherapy, were provided in the event of documented relapse or persistent disease.

\section{Follow-up and statistical analyses}

The duration of patient follow-up was calculated from the first day of treatment to either the day of death or the day of the last examination. The patients were examined at least every 3 months during the first 2 years; thereafter, a follow-up examination was performed every 6 months for up to 5 years or until death. The time of last follow-up was March 2010, and the median followup period was 61.4 months (range, 2.5-86.6 months).

All events were measured from the date of treatment commencement. The following end points (time to the first defining event) were assessed: overall survival (OS); disease-specific survival (DSS); local relapse-free survival (LRFS); distant metastasis-free survival (DMFS); and disease-free survival (DFS). In calculating the diseasespecific survival, a patient dying of their tumour was a point event; patients who survived, defaulted follow-up, or died of other disease were censored. Local recurrence was established by fibreoptic endoscopy and biopsy or MRI. Distant metastases were diagnosed on the basis of clinical symptoms, physical examination, and imaging methods, including chest radiography, bone scans, MRI and abdominal sonography.

All statistical analyses were performed using the Statistical Package for the Social Sciences (SPSS) 13.0 software (SPSS Inc., Chicago, IL). The actuarial rates were calculated by the Kaplan-Meier method, and differences were compared using the log-rank test. Multivariate analyses with the Cox proportional hazards model were used to calculate the hazard ratio (HR) and test independent significance by backward elimination of insignificant explanatory variables. Host factors (age and sex) were included as the covariates in all tests. The criterion for significance was set at 0.05 , and $\mathrm{P}$ values were based on 2 -sided test results.

\section{Results}

\section{Incidence and Survival of CN Palsy}

Of the 1892 patients with NPC, 178 (9.4\%) had clinical evidence of $\mathrm{CN}$ palsy at presentation including 171 patients with unilateral palsy and 7 with bilateral palsy. The most common $\mathrm{CN}$ palsies among these patients involved VI, V2 and V3 (49.4\%, $44.9 \%$ and $35.4 \%$, respectively). In $178 \mathrm{~T}_{4}$ patients, the 5-year survival rates were as follows: overall survival (OS), 61.0\%; diseasespecific survival (DSS), 69.6\%; local relapse-free survival (LRFS), 75.2\%; distant metastasis-free survival (DMFS), 73.4\%; and disease-free survival (DFS), $55.3 \%$.

\section{Differences in the prognostic implications of single and multiple $\mathrm{CN}$ palsy}

Of the 178 patients, 74 patients $(41.6 \%)$ had single $\mathrm{CN}$ palsy and 104 patients (58.4\%) had multiple CN palsy. Significant differences were observed in the 5-year OS rates $(69.8 \%$ vs. $54.3 \% ; \mathrm{P}=0.033)$ between the two groups with single and multiple CN palsy, but no significant differences were observed for DSS (76.1\% vs. 64.5\%; $\mathrm{P}=0.085)$, LRFS $(73.2 \%$ vs. $76.8 \% ; \mathrm{P}=0.783)$, DMFS ( $76.9 \%$ vs. $70.5 \% ; \mathrm{P}=0.384)$ or $\mathrm{DFS}(59.0 \%$ vs. $52.7 \%$; $\mathrm{P}=0.317$ ) rates between the two groups (Figure 1).

The following parameters, which could potentially influence patient prognosis, were included in the Cox proportional hazards model for multivariate analysis: age (>median age vs. $\leq$ median age), sex, $\mathrm{N}$ classification, radiotherapy dose (>70 Gy vs. $\leq 70$ Gy), chemotherapy, and $\mathrm{CN}$ palsy (with single and multiple involvement). The results indicated that the number of $\mathrm{CN}$ palsies was an independent prognostic factor for OS $(\mathrm{HR}=1.765$; $\mathrm{P}=0.029)$, but not for DSS, LRFS, DMFS or DFS.

\section{Prognosis of upper and lower $\mathrm{CN}$ palsy}

Of the 178 patients, 140 patients (78.7\%) had upper $\mathrm{CN}$ palsy and 17 patients $(9.6 \%)$ had lower $\mathrm{CN}$ palsy. No significant differences were observed for OS $(61.2 \%$ vs. $67.7 \% ; \mathrm{P}=0.581)$, DSS (70.1\% vs. $72.2 \% ; \mathrm{P}=0.792)$, LRFS (75.4\% vs. $73.9 \% ; \mathrm{P}=0.729)$, DMFS $(70.1 \%$ vs. $86.5 \%$; $\mathrm{P}=0.257)$ or DFS $(52.7 \%$ vs. $73.9 \% ; \mathrm{P}=0.212)$ rates between the two groups (Figure 2).

The following parameters, which could potentially influence patient prognosis, were included in the Cox proportional hazards model for multivariate analysis: age (>median age vs. $\leq$ median age), sex, $\mathrm{N}$ classification, radiotherapy dose (>70 Gy vs. $\leq 70$ Gy), chemotherapy, and $\mathrm{CN}$ palsy (with upper and lower involvement). The results indicated that the location of $\mathrm{CN}$ palsy was not an independent prognostic factor for OS, DSS, LRFS, DMFS or DFS.

\section{Prognostic value of different pretreatment durations}

Because the patients with $\mathrm{CN}$ palsy had different duration times before clinical diagnosis, it is essential to determine the prognostic value of the pretreatment duration. The patients with $\mathrm{CN}$ palsy in this series were classified into two groups: Group 1 included patients with $\mathrm{CN}$ palsy with a pretreatment duration within 2 months, and Group 2 had a pretreatment duration longer than 2 months. A significant difference was observed for OS $(68.7 \%$ vs. $43.3 \% ; \mathrm{P}=0.007)$ between Groups 1 and 2, but no significant differences were observed for DSS $(74.0 \%$ vs. $56.8 \%$; $\mathrm{P}=0.222)$, LRFS (78.9\% vs. $66.8 \% ; \mathrm{P}=0.068)$, DMFS $(72.4 \%$ vs. $74.7 \%$; $\mathrm{P}=0.532)$ or DFS $(57.5 \%$ vs. $49.7 \%$; $\mathrm{P}=0.369)$ rates between the two groups (Figure 3). 

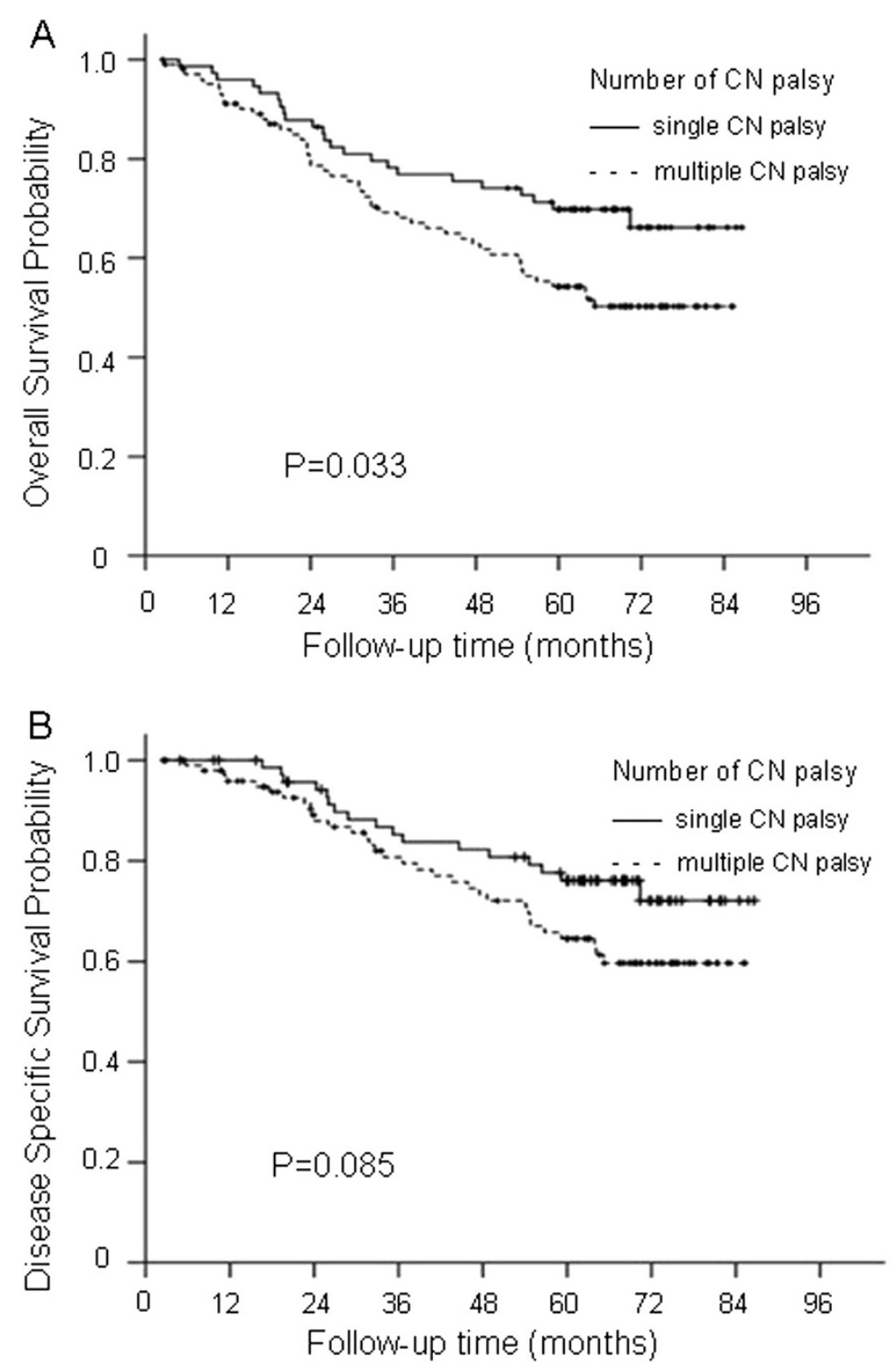

Figure 1 The overall survival and disease-specific survival curves for the different numbers of CN palsy groups: Group 1, patients with single CN palsy; Group 2, patients with multiple CN palsy (A and B).

A multivariate analysis was performed to adjust for various prognostic factors in Group 1 and 2 patients with different pretreatment durations of $\mathrm{CN}$ palsy. The following parameters were included in the Cox proportional hazards model by backward elimination of insignificant explanatory variables: age (>median age vs. $\leq$ median age), sex, $\mathrm{N}$ classification, radiotherapy dose (>70 Gy vs. $\leq 70$ Gy), chemotherapy, and pretreatment duration (Groups 1 and 2). The results indicated that pretreatment duration was an independent prognostic factor for OS $(H R=1.926 ; \mathrm{P}=0.009)$ but not for DSS, LRFS, DMFS or DFS.

\section{Further prognostic analysis of patients with different} recovery durations

Because most patients recover from $\mathrm{CN}$ palsy within six months, all patients with $\mathrm{CN}$ palsy in this series were classified into two groups: Group 1 included patients with a recovery duration within 6 months, and Group 2 included patients with a recovery duration beyond 6 months. Significant differences were observed for 5 -year OS (69.1\% vs. 41.7\%; $\mathrm{P}<0.001)$, DSS $(75.8 \%$ vs. $54.4 \% ; \mathrm{P}=0.016)$, LRFS $(82.3 \%$ vs. $59.5 \% ; \mathrm{P}=0.002)$ and DFS ( $63.9 \%$ vs. $36.4 \%$; $\mathrm{P}=0.001)$ rates between Groups 1 and 2, but no significant difference was observed for 

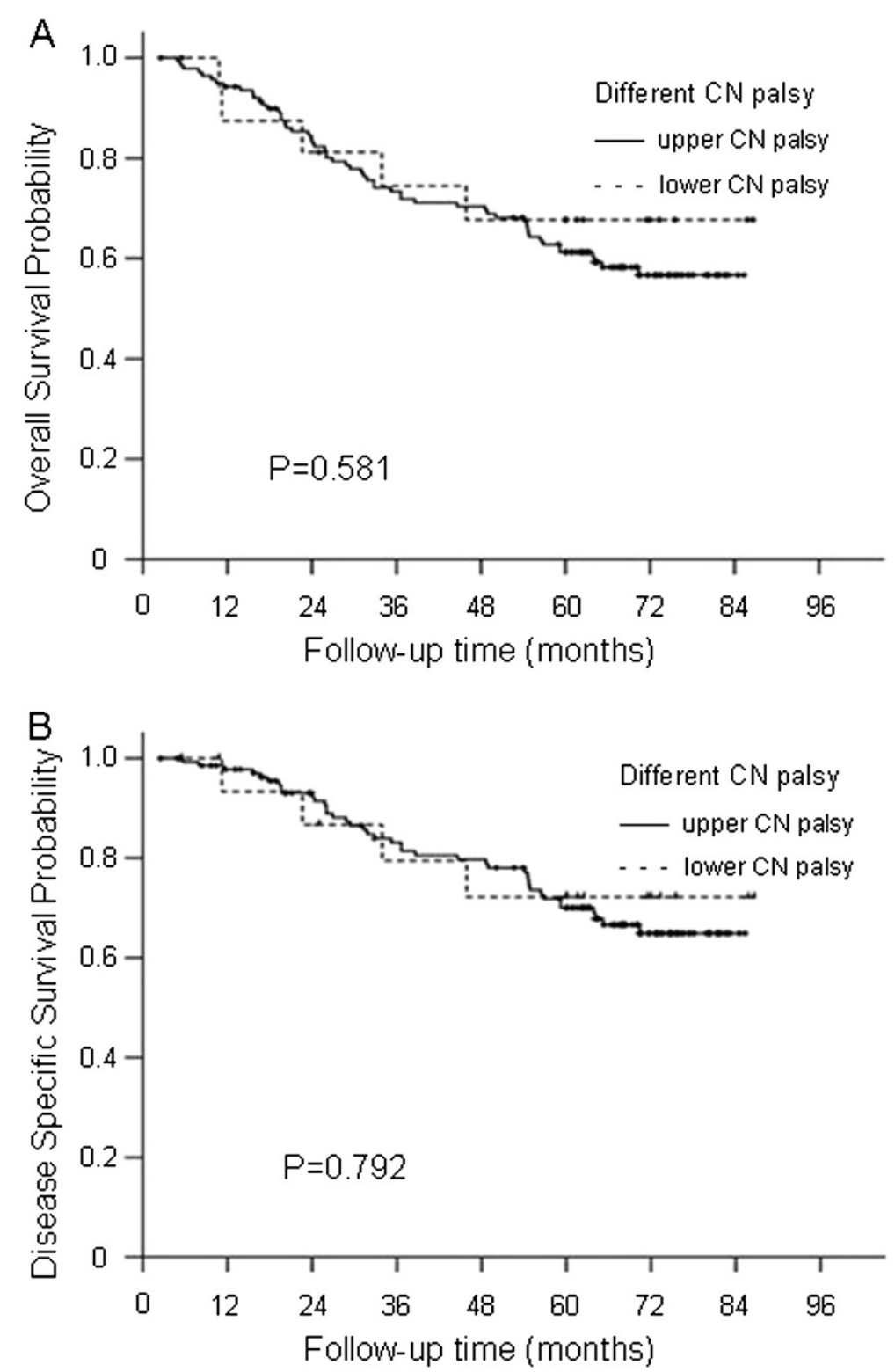

Figure 2 The overall survival and disease-specific survival curves for the different section groups: Group 1, patients with upper CN palsy; Group 2, patients with lower CN palsy (A and B).

DMFS (75.6\% vs. 66.8\%; P $=0.405)$ rates between Groups 1 and 2 (Figure 4).

A multivariate analysis was performed to adjust for various prognostic factors in Group 1 and 2 patients with different recovery durations of $\mathrm{CN}$ palsy. The following parameters were included in the Cox proportional hazards model by backward elimination of insignificant explanatory variables: age (>median age vs. $\leq$ median age), sex, $\mathrm{N}$ classification, radiotherapy dose (>70 Gy vs. $\leq 70$ Gy), chemotherapy, and recovery duration (Groups 1 and 2). The results indicated that recovery duration was an independent prognostic factor for OS $(\mathrm{HR}=2.485 ; \mathrm{P}<0.001)$, DSS $(\mathrm{HR}=2.065 ; \mathrm{P}=0.016)$,
LRFS $\quad(\mathrm{HR}=3.051 ; \quad \mathrm{P}=0.001) \quad$ and $\mathrm{DFS} \quad(\mathrm{HR}=2.440$; $\mathrm{P}<0.001)$ but not for DMFS.

\section{Discussion}

In this study, the incidence of cranial nerve palsy (9.4\%) was similar to that found in previous research (approximately 8.0 to $12.4 \%$ ) [1]. The AJCC has recommended that $\mathrm{CN}$ involvement be assessed through neurological evaluation rather than cross-sectional imaging [2]. Because $\mathrm{CN}$ involvement is considered a poor prognostic indicator in NPC patients, those with $\mathrm{CN}$ involvement are staged as T4. CN palsy frequently accompanied skull-base invasion in upper $\mathrm{CN}$ palsy and carotid sheath 

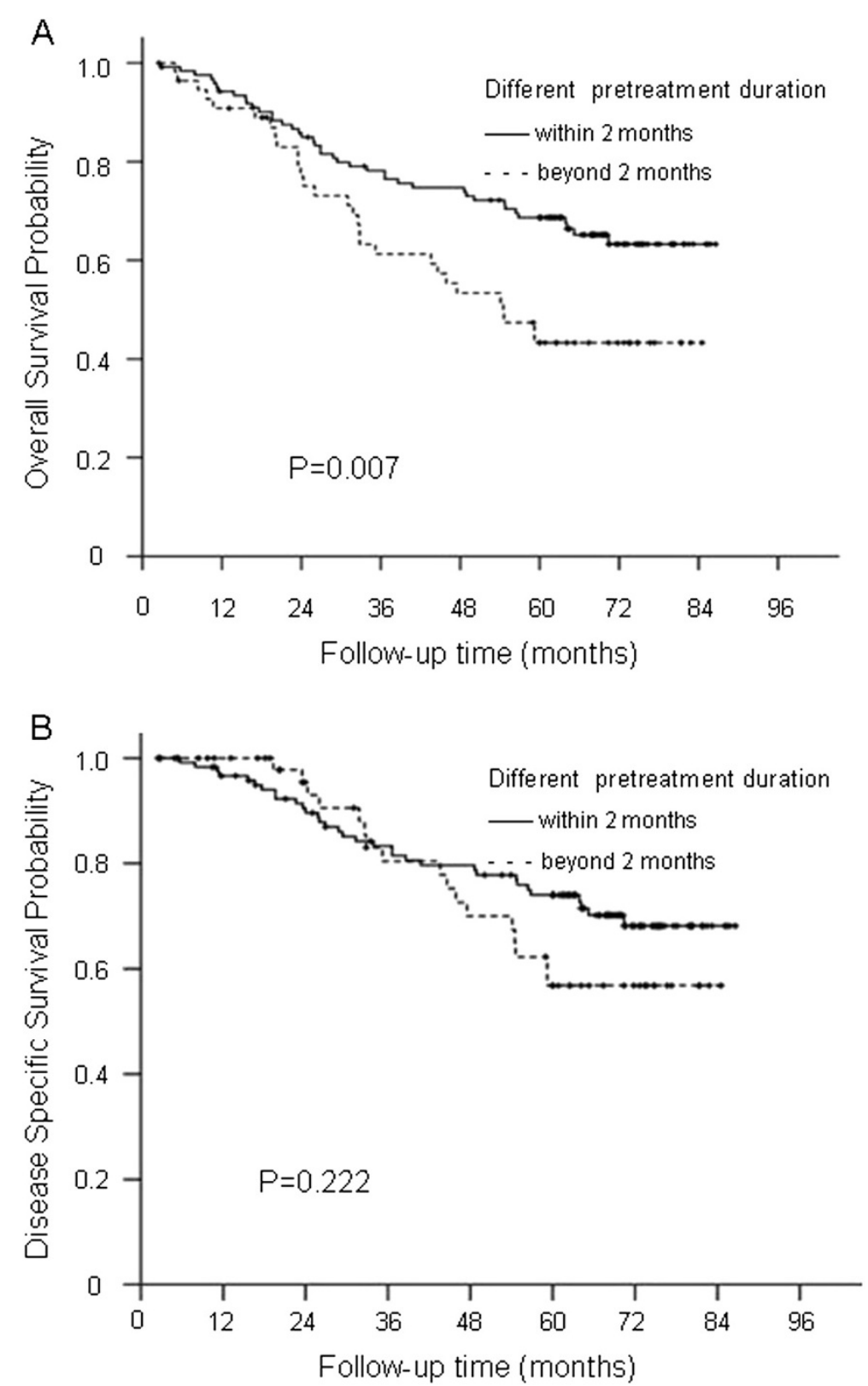

Figure 3 The overall survival and disease-specific survival curves for the different pretreatment duration groups: Group 1, patients with CN palsy with a pretreatment duration within 2 months; Group 2, patients with a pretreatment duration beyond 2 months ( $A$ and $B)$.

erosion in lower CN palsy. Moreover, skull-base invasion frequently approaches from basicranial to intracranial or orbital sites and involves upper CNs. In addition, the $\mathrm{CN}$ $\mathrm{V}$ and VI are located in the sites most prone to tumor invasion and extend a long distance; their involvement can often be observed.

The results of this study showed that NPC patients with multiple $\mathrm{CN}$ palsy have poorer OS than those with single $\mathrm{CN}$ palsy, but the difference in disease-specific survival rates was not significant. Patient prognosis is likely related to the extent and volume of the tumor, and the incorporation of the primary tumor volume may lead to a further refinement of treatment outcome $[3,11]$. $\mathrm{NPC}$ patients with multiple $\mathrm{CN}$ palsy may require more cycles of induction or adjuvant chemotherapy in addition to the base of concomitant chemotherapy and radiation [12,13]. However, the results of our study revealed that the DSS was not significant for different groups with single and multiple $\mathrm{CN}$ palsy, which may account for the observation that different numbers of 

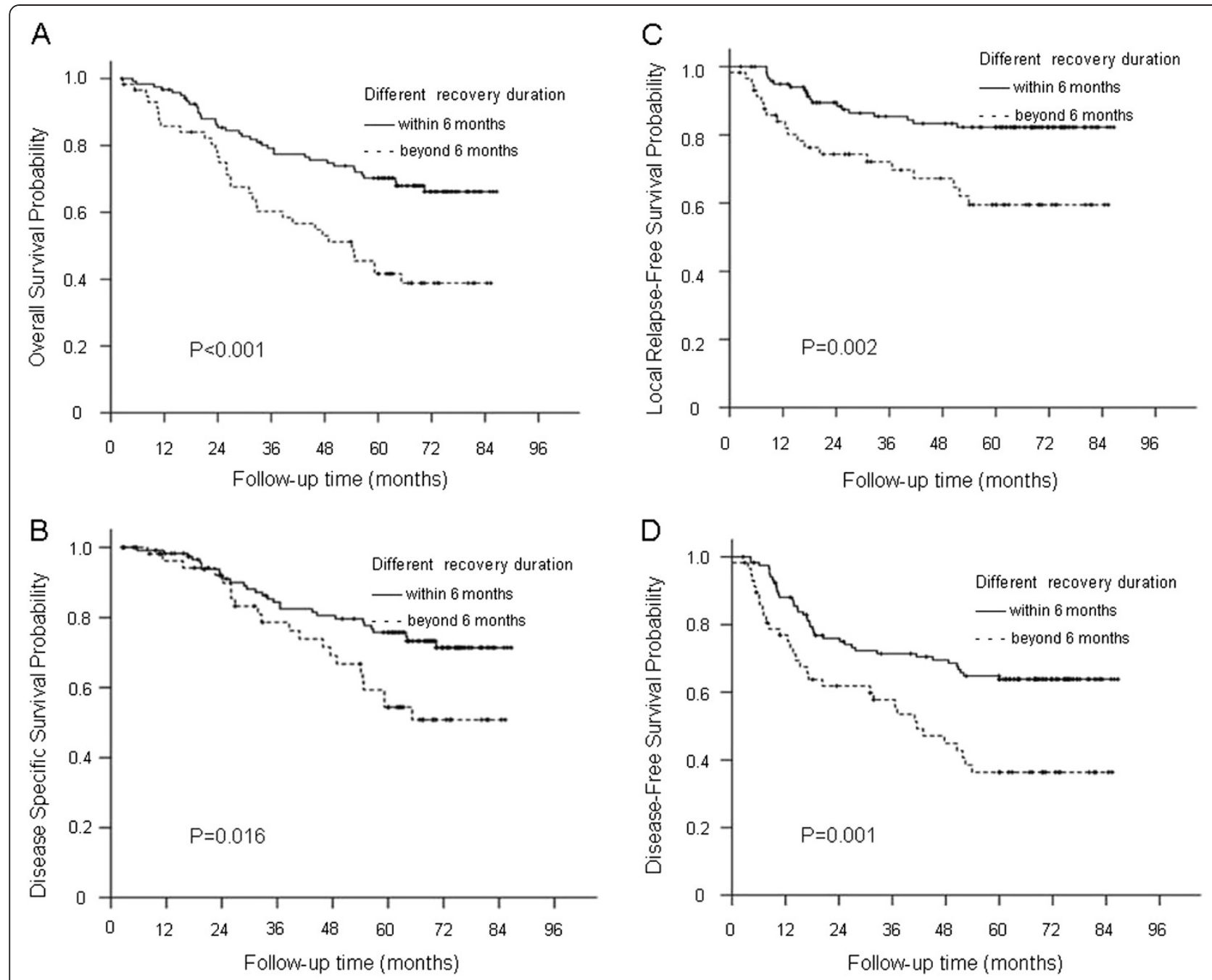

Figure 4 The overall survival, disease-specific survival, local relapse-free survival and disease-free survival curves for the different recovery duration groups: Group 1, recovery duration of patients with $\mathrm{CN}$ palsy within 6 months; Group 2, recovery duration beyond 6 months $(A / B / C / D)$.

$\mathrm{CN}$ palsy were not the crucial cause of death. Moreover, $\mathrm{CN}$ palsy with upper or lower involvement was not found to be a significant factor influencing OS, DSS, LRFS or DFS. This result was likely related to anatomy. $\mathrm{CN}$ palsy often accompanies basicranial, intracranial or orbital invasion in upper $\mathrm{CN}$ palsy and carotid sheath erosion in lower $\mathrm{CN}$ palsy. Upper $\mathrm{CN}$ involvement was associated with recurrence from the primary invasion; in contrast, lower $\mathrm{CN}$ involvement accompanied lymph node erosion, which could increase metastasis, exhibiting the hazard criteria consistent with the staging classification system [14]. Advanced $\mathrm{T}$ and $\mathrm{N}$ stages had similar prognoses.

In 2010, the NCCN recommended that the treatment regimen for NPC patients with $\mathrm{CN}$ involvement should consist of concurrent chemoradiotherapy plus adjuvant or induction chemotherapy as the standard treatment.
Despite the fact that the pretreatment duration was associated with significantly poorer OS in this study, the difference in DSS was not significant between the groups with different pretreatment durations. Moreover, no other study has assessed the relationship between pretreatment duration and prognosis. Further studies are required to determine whether more regimens and cycles of chemotherapy would add a benefit to radiotherapy for patients with different pretreatment durations. These findings may have implications for further improvements in treating advanced-stage tumors with different pretreatment durations.

In our study, recovery duration was an independent prognostic factor for 5-year LRFS in patients with NPC. In a previous study, $\mathrm{CN}$ recovery correlated significantly with the pretreatment duration of $\mathrm{CN}$ palsy, the time course of clinical tumor regression, and neurologic 
symptom improvement during radiation [15], which recommended a need for continuous and close neurologic surveillance. In addition, patients with NPC had a higher rate of radiation-induced $\mathrm{CN}$ palsy, with a median latent period of 7.6 years (range, 0.3-34 years) [16]. It is essential to examine $\mathrm{CN}$ involvement before treatment and to estimate the cause from clinical data; in particular, MRI has been shown to be a valuable tool for detecting and defining the extent of $\mathrm{CN}$ involvement in NPC [17]. The results of our study showed that patients whose recoveries from $\mathrm{CN}$ palsy required longer than six months had poorer OS, DSS, LRFS and DFS. We hypothesize that recovery duration is related to recurrence, which leads to poor survival. Thus, patients with longer recovery durations require follow-up after six months to detect recurrence and sequelae that influence patient prognosis.

\section{Conclusions}

In conclusion, recovery duration is an independent prognostic factor for NPC with $\mathrm{CN}$ palsy and is related to recurrence that leads to poor survival. Moreover, recovery duration requires close surveillance and different treatment regimens.

\section{Abbreviations}

AJCC: American joint committee on cancer; NCCN: National comprehensive cancer network; NPC: Nasopharyngeal carcinoma; CN: Cranial nerve; OS: Overall survival; DSS: Disease-specific survival; LRFS: Local relapse-free survival; DMFS: Distant metastasis-free survival; DFS: Disease-free survival.

\section{Competing interests}

The authors declare that they have no competing interests.

\section{Authors' contributions}

Guarantors of integrity of the entire study: H-YM, RS, H-QM; study concepts/ study design, data acquisition or data analysis/interpretation: all authors; manuscript drafting or revision for important intellectual content: all authors; manuscript final version approval: all authors; literature review: R. Sun, $\mathrm{H}-\mathrm{Q}$ Mai; clinical studies: RS, W-JH, Y-XL, JY; statistical analyses: RS, JS, QZ, W-JH; and manuscript editing: RS, JS, QZ.

\section{Authors' information \\ H-Y Mo (first author) \\ R. Sun (co-first author)}

\section{Acknowledgments}

This work was supported by grants from the National Natural Science Foundation of China (No: 30600755; No: 81072226), the 863 Project (No: 2012AA02A501), the Sci-Tech Project Foundation of Guangdong Province (No: 2011B080701034), the Sci-Tech Project Foundation of Guangzhou City (No: 2011 J4300100), and Fundamental Research Funds for the Central Universities.

\section{Author details}

${ }^{1}$ State Key Laboratory of Oncology in South China, Sun Yat-sen University Cancer Center, No, 651 Dongfeng Road East, Guangzhou 510060, People's Republic of China. ${ }^{2}$ Department of Nasopharyngeal Carcinoma, Sun Yat-sen University Cancer Center, 651 Dongfeng Road East, Guangzhou 510060, People's Republic of China. ${ }^{3}$ Department of Clinical Trial Center, Sun Yat-sen University Cancer Center, No, 651 Dongfeng Road East, Guangzhou 510060, People's Republic of China. ${ }^{4}$ Key Laboratory of Gene Engineering of the Ministry of Education, School of Life Sciences, Sun Yat-sen University, No, 135 Xingang Road West, Guangzhou 510275, People's Republic of China.
${ }^{5}$ Department of Radiotherapy, Affiliated Cancer Hospital, Guangzhou Medical College, No, 78 Luhu Road, Guangzhou 510095, People's Republic of China.

Received: 12 April 2012 Accepted: 4 September 2012

Published: 7 September 2012

\section{References}

1. Cheng SH, Tsai SY, Horng CF, et al: A prognostic scoring system for locoregional control in nasopharyngeal carcinoma following conformal radiotherapy. Int J Radiat Oncol Biol Phys 2006, 66(4):992-1003.

2. Edge SB, Byrd DR, Compton CC, et al: American Joint Committee on Cancer Staging Manual. New York: Seventh Edition; 2009.

3. Chang JT, Lin CY, Chen TM, et al: Nasopharyngeal carcinoma with cranial nerve palsy: the importance of MRI for radiotherapy. Int J Radiat Oncol Biol Phys 2005, 63(5):1354-1360.

4. Su CY, Lui CC: Perineural invasion of the trigeminal nerve in patients with nasopharyngeal carcinoma Imaging and clinical correlations. Cancer 1996, 78(10):2063-2069.

5. Chong VF, Fan YF: Pterygopalatine fossa and maxillary nerve infiltration in nasopharyngeal carcinoma. Head Neck 1997, 19(2):121-125.

6. Chen L, Liu LZ, Mao YP, et al: Grading of MRI-detected skull-base invasion in nasopharyngeal carcinoma and its prognostic value. Head Neck 2011, 33(9):1309-1314

7. Baujat B, Audry H, Bourhis J, et al: Chemotherapy in locally advanced nasopharyngeal carcinoma: an individual patient data meta-analysis of eight randomized trials and 1753 patients. Int J Radiat Oncol Biol Phys 2006, 64(1):47-56.

8. Ma J, Liu L, Tang $L$, et al: Retropharyngeal lymph node metastasis in nasopharyngeal carcinoma: prognostic value and staging categories. Clin Cancer Res 2007, 13(5):1445-1452.

9. Zhao C, Han F, Lu LX, et al: Intensity modulated radiotherapy for localregional advanced nasopharyngeal carcinoma [Article in Chinese]. Ai Zheng 2004, 23(11 Suppl):1532-1537.

10. Luo W, Deng XW, Lu TX: Dosimetric evaluation for three dimensional conformal, conventional, and traditional radiotherapy plans for patients with early nasopharyngeal carcinoma [Article in Chinese]. Ai Zheng 2004 23(5):605-608

11. Chen MK, Chen TH, Liu JP, Chang CC, Chie WC: Better prediction of prognosis for patients with nasopharyngeal carcinoma using primary tumor volume. Cancer 2004, 100(10):2160-2166.

12. Chi KH, Chang YC, Guo WY, et al: A phase III study of adjuvant chemotherapy in advanced nasopharyngeal carcinoma patients. Int J Radiat Oncol Biol Phys 2002, 52(5):1238-1244.

13. Lin JC, Jan JS, Hsu CY, Liang WM, Jiang RS, Wang WY: Phase III study of concurrent chemoradiotherapy versus radiotherapy alone for advanced nasopharyngeal carcinoma: positive effect on overall and progressionfree survival. J Clin Oncol 2003, 21(4):631-637.

14. Groome PA, Schulze K, Boysen M, Hall SF, Mackillop WJ: A comparison of published head and neck stage groupings in carcinomas of the oral cavity. Head Neck 2001, 23(8):613-624.

15. Li JC, Mayr NA, Yuh WT, Wang JZ, Jiang GL: Cranial nerve involvement in nasopharyngeal carcinoma: response to radiotherapy and its clinical impact. Ann Otol Rhinol Laryngol 2006, 115(5):340-345.

16. Kong L, Lu JJ, Liss AL, Hu C, Guo X, Wu Y, Zhang Y: Radiation-induced cranial nerve palsy: a cross-sectional study of nasopharyngeal cancer patients after definitive radiotherapy. Int J Radiat Oncol Biol Phys 2011, 79(5):1421-1427.

17. Liu L, Liang S, Li L, et al: Prognostic impact of magnetic resonance imaging-detected cranial nerve involvement in nasopharyngeal carcinoma. Cancer 2009, 115(9):1995-2003.

doi:10.1186/1748-717X-7-149

Cite this article as: Mo et al.: Prognostic value of pretreatment and recovery duration of cranial nerve palsy in nasopharyngeal carcinoma. Radiation Oncology 2012 7:149. 